\title{
Mechanisms of Anomalous Dispersion in Flow Through Heterogeneous Porous Media
}

\author{
Alina Tyukhova, ${ }^{1,2}$ Marco Dentz, ${ }^{3, *}$ Wolfgang Kinzelbach, ${ }^{1}$ and Matthias Willmann ${ }^{1}$ \\ ${ }^{1}$ Institute of Environmental Engineering, ETH Zurich, Zurich, Switzerland. \\ ${ }^{2}$ Department of Civil and Environmental Engineering, \\ Massachusetts Institute of Technology, Cambridge, MA, USA \\ ${ }^{3}$ Spanish National Research Council (IDAEA-CSIC), Barcelona, Spain
}

(Dated: October 19, 2016)

\begin{abstract}
We study the origins of anomalous dispersion in heterogeneous porous media in terms of the medium and flow properties. To identify and quantify the heterogeneity controls, we focus on porous media which are organized in assemblies of equally sized conductive inclusions embedded in a constant conductivity matrix. We study the behavior of particle arrival times for different conductivity distributions and link the statistical medium characteristics to large scale transport using a continuous time random walk (CTRW) approach. The CTRW models particle motion as a sequence of transitions in space and time. We derive an explicit map of the conductivity onto the transition time distribution. The derived CTRW model predicts solute transport based on the conductivity distribution and the characteristic heterogeneity length. In this way, heavy tails in solute arrival times and anomalous particle dispersion as measured by the centered mean square displacement are directly related to the medium properties. These findings shed light on the mechanisms of anomalous dispersion in heterogeneous porous media, and provide a basis for the predictive modeling of large scale transport.
\end{abstract}

\section{INTRODUCTION}

Anomalous dispersion has been widely observed in transport through heterogeneous porous media [1-5]. It manifests itself in heavy tails in solute arrival time distributions, or breakthrough and the non-linear evolution of the second centered moments of solute distributions. Anomalous dispersion can be caused by different physical processes, chemical heterogeneity $[6,7]$, the interplay of physical heterogeneity and diffusion [8,9], and physical heterogeneity alone. Here, we concentrate on the impact of physical heterogeneity in the distribution of hydraulic conductivity $[4,5,10,11]$. In highly heterogeneous fields fast flow concentrates along highly permeable preferential paths consisting of connected structures of large hydraulic conductivity[12-14]. The spatial complement to the flow channels form disconnected zones of slow advective velocities where solutes are delayed. The interplay of fast channels and slow advection in disconnected zones leads to anomalous dispersion.

The impact of these mechanisms on large scale transport can be described in terms of continuous times random walks (CTRW). The CTRW $[15,16]$ has found applications for the modeling of anomalous diffusion in a wide range of physical systems [2, 17-20]. Berkowitz and Scher $[2,21]$, have realized that the CTRW provides the dynamics needed to characterize non-Fickian hydrodynamic transport in heterogeneous porous and fractured media. The CTRW describes particle movements as a random walk in space and time as $[9,22]$

$$
x_{n+1}=x_{n}+\ell_{n}, \quad t_{n+1}=t_{n}+\frac{\ell_{n}}{v_{n}},
$$

with $\ell_{n}$ the transition length and $v_{n}$ the particle velocity. The spatial jumps and waiting times may be independent or correlated random variables [23]. The transition times in the CTRW are given in terms of the particle velocities $v_{n}$, whose statistics have typically been estimated by using particle tracking simulations in the detailed heterogeneous flow [23-26]. The multirate mass transfer (MRMT) framework models the interplay of fast channels and slow advection by a mobile-immobile approach. Fast channels define the mobile zone, regions of slow advection are represented as immobile. The mobile and immobile regions are connected through linear mass transfer [27-29] characterized by the memory function. The latter encodes the distribution of residence times in the immobile zones, which in principle is related to the statistics of slow advection. CTRW and MRMT have a similar phenomenological basis and both model history dependent transport dynamics. In fact, it has been shown [30-32] that both models are under certain conditions mathematically equivalent. For both modeling approaches, the relation between the (statistical) medium and flow properties and large scale transport is of central importance [2, 26, 33-35]. Oftentimes, the distribution of transition times (CTRW) and the memory function (MRMT) are estimated on the basis of coupled flow and transport simulations or from experimental data, for example breakthrough curves. For some systems such as diffusion in quenched random trap models [36], or hydrodynamic transport under linear retention due to physical and chemical medium heterogeneity, can the medium properties and geometry be directly linked to the average non-Fickian transport behavior [37-39]. The objective here is to investigate the quantitative link between the hydraulic conductivity distribution and large scale transport in the framework of CTRW and thus elucidate the heterogeneity controls on anomalous dispersion. 
To this end, we consider transport in the flow through heterogeneous porous media which are organized in assemblies of equally sized conductive inclusions embedded in a constant conductivity matrix. The conductivities inside the inclusions are constant and distributed between the inclusions. This type of media serves as models for heterogeneous porous media characterized by finite correlation length and arbitrary conductivity point distributions. Eames and Bush [40] studied solute dispersion in such media and derived expressions for the dispersion coefficients. Fiori et al. [41, 42, 43] studied anomalous transport in media consisting of inclusions with lognormal distributions of hydraulic conductivity and derived semi-analytical expressions for solute travel times. These solutions have been implemented into a time-domain random walk approach, which is similar to the CTRW [44].

In this paper we investigate anomalous transport and its heterogeneity controls through detailed numerical simulations of flow and particle transport in realizations of the model porous medium. The next section introduces the flow and transport model, defines the heterogeneous model medium, and discusses the flow properties. Section III derives the CTRW model to quantify anomalous dispersion, and the relations between the transition time distribution, particle and flow velocities, and the hydraulic conductivity distribution. Section IV applies the developed model to predict first passage time distributions obtained from direct numerical simulations of flow and particle transport in realization of the heterogeneous model media for power-law and lognormal distributions of hydraulic conductivity. Section V uses the developed CTRW approach to study the dispersion properties in highly heterogeneous porous media.

\section{FLOW AND TRANSPORT MODEL}

In this section we outline the transport and flow models and define the heterogeneous porous medium model under consideration.

\section{A. Transport}

We consider particle transport in the absence of microscale dispersion and focus solely on the impact of heterogeneous advection on the dispersion of an advected scalar $c(\mathbf{x}, t)$. Its evolution in a divergence-free flow $\mathbf{v}(\mathbf{x})$ with $\nabla \cdot \mathbf{v}(\mathbf{x})=0$ is governed by the Liouville equation

$$
\frac{\partial c(\mathbf{x}, t)}{\partial t}=-\mathbf{v}(\mathbf{x}) \cdot \nabla c(\mathbf{x}, t)
$$

The coordinate vector is $\mathbf{x}=(x, y)^{\top}$, where the superscript $T$ denotes the transpose. We take a Lagrangian view point in order to derive the effective equations of motion of the dispersed scalar and start from the equiv-

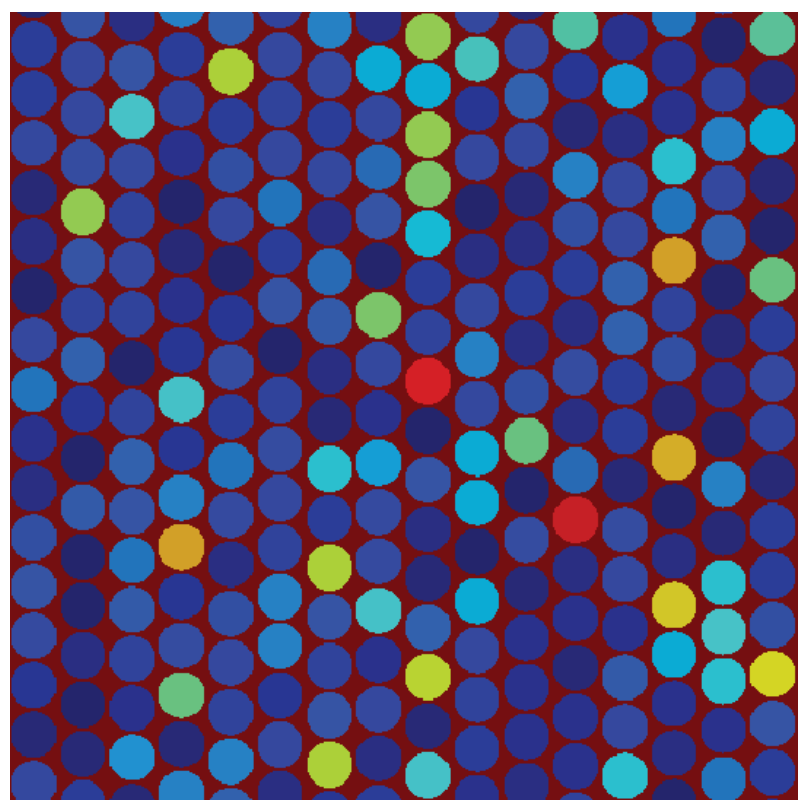

FIG. 1. Illustration of the model medium consisting of a conductive matrix with $k_{0}=1$ and less conductive disc-shaped inclusions of radius $r_{0}=1$ and conductivity $k$, which is distributed according to $p_{k}(k)$. The inclusions are embedded in a rectangular unit cell of size $\ell_{0}$. Different colors denote different conductivity values.

alent advection equation

$$
\frac{d \mathbf{x}(t)}{d t}=\mathbf{v}[\mathbf{x}(t)]
$$

which describes the evolution of scalar particles, whose density is denoted by $c(\mathbf{x}, t)$. We employ a stochastic framework to quantify the average transport behavior in the heterogeneous flow field $\mathbf{v}(\mathbf{x})$. This means $\mathbf{v}(\mathbf{x})$ is considered a realization of an ensemble of random flow fields whose statistical properties are discussed in the next section.

In this framework, we study the ensemble transport behavior in terms of the first passage times at a plane located at $x=x_{c}$,

$$
\tau\left(x_{c}\right)=\inf \left\{t \mid x(t) \geq x_{c}\right\}
$$

The probability density function (PDF) of first passage times is given by

$$
f\left(t, x_{c}\right)=\left\langle\delta\left[t-\tau\left(x_{c}\right)\right]\right\rangle
$$

where the angular brackets denote the combined average over all particles launched in a given realization and the average over the ensemble of random media. Note that $f\left(t, x_{c}\right)$ is equivalent to the solute breakthrough curve measured at the position $x_{c}$. 


\section{B. Flow}

We consider here flow through heterogeneous porous media that are composed of a homogeneous matrix of hydraulic conductivity $k_{0}$ and equally sized discs of radius $r_{0}$. Each sphere is embedded in a unit cell of length $\ell_{0}$. The conductivities $k$ of the disc-shaped inclusions are assigned randomly from the PDF $p_{k}(k)$. Without loss of generality, we set $k_{0}=1$ and $r_{0}=1$ in the following. The volume fractions of the disc-shaped inclusions is given by $\chi=\pi / \ell_{0}^{2}$. A realization of the random media under consideration is illustrated in Figure 1.

The flow velocity $\mathbf{v}(\mathbf{x})$ through this medium is given by the Darcy equation [45],

$$
\mathbf{v}(\mathbf{x})=-k(\mathbf{x}) \nabla h(\mathbf{x})
$$

where $k(\mathbf{x})$ denotes hydraulic conductivity and $h(\mathbf{x})$ hydraulic head. As outlined in the following, hydraulic conductivity is modeled as a spatial random field, this means $\mathbf{v}(\mathbf{x})$ is a realization of an ensemble of random flow fields characterized by certain statistical properties. Flow is driven here by a uniform mean hydraulic gradient $\nabla \bar{h}(\mathbf{x})$ that is aligned with the $x$-axis of the coordinate system, see also Appendix A 1.

\section{Single Inclusion}

In order to characterize the flow in the random medium, we first consider flow through an isolated unit cell embedded in an infinite porous matrix. The steadystate flow potential function is given by $[40,46]$

$$
\Phi(\mathbf{r})=u_{m}\left(1+\frac{1-k}{1+k} \frac{1}{r^{2}}\right) r_{1}
$$

for $r \equiv|\mathbf{r}|>1$ and

$$
\Phi(\mathbf{r})=\frac{2 u_{m} k r_{1}}{1+k}
$$

for $r \leq 1$. Note that $\mathbf{r}=\left(r_{1}, r_{2}\right)^{\top}$ refers to the coordinate system with the origin in the center of the circular inclusion; $u_{m}$ denotes the velocity in the matrix at infinity. The velocity field is given by $\mathbf{u}(\mathbf{r})=\nabla \Phi(\mathbf{r})$. In the matrix outside the inclusion, we have

$$
\begin{aligned}
\mathbf{u}_{o}(\mathbf{r}) & =u_{m}\left(1+\frac{1-k}{1+k} \frac{r_{2}^{2}-r_{1}^{2}}{r^{4}}\right) \mathbf{e}_{1} \\
& -u_{m} \frac{1-k}{1+k} \frac{2 r_{1} r_{2}}{r^{4}} \mathbf{e}_{2}
\end{aligned}
$$

for $r>1$. Inside the inclusion, the flow velocity is constant and given by

$$
\mathbf{u}_{i}(\mathbf{r})=\frac{2 u_{m} k}{1+k} \mathbf{e}_{1}
$$

for $r \leq 1$ with $\mathbf{e}_{i}$ the unit vector aligned with the $i-$ direction of the coordinate system. Darcy velocities inside the inclusion are uniform and aligned with the direction of the mean pressure gradient.

\section{Distribution of Inclusions}

We consider now the properties of the flow in the medium illustrated in Figure 1. The conductivities within the inclusions are drawn independently from the distribution $p_{k}(k)$. In order to transfer the information on the flow for the single inclusion to the random medium, some remarks are in order. As the flow potential (7) decreases as $r^{-2}$, we assume that interaction between the discs can be neglected. Thus, in the following, we use expressions (8a) and (8b) as an approximation for the velocity field in the unit cell. The characteristic matrix velocity $u_{m}$ and the effective background conductivity $k^{e}$ are still to be determined. They are imposed by the boundary conditions and the medium geometry. In order to determine $k^{e}$, we consider the average flow velocity $\bar{q}$, which is given by the effective Darcy equation $[47,48]$

$$
\bar{q}=-k^{e}|\nabla \bar{h}|,
$$

where $k^{e}$ is the effective conductivity of the medium and $\nabla \bar{h}$ is the hydraulic gradient, which here is aligned with the one-direction of the coordinate system. The Maxwell formula gives for $k^{e}[49,50]$

$$
k^{e}=\frac{1+\chi \Lambda}{1-\chi \Lambda}, \quad \Lambda=\int_{0}^{\infty} d k p_{k}(k) \frac{k-1}{k+1},
$$

where $\chi=\frac{\pi r^{2}}{\ell^{2}}$ is the volume fraction of the inclusions. For strong conductivity contrasts between the inclusions and the matrix, that is $\langle k\rangle \ll 1$ we may approximate $\Lambda \approx-1$. In this case the effective conductivity is

$$
k^{e} \approx \frac{1-\chi}{1+\chi}
$$

In general, we evaluate the integral in (10) using the full conductivity distribution $p_{k}(k)$.

As outlined in Ref. [49], Maxwell's approximation gives good estimates for $k^{e}$ also for non-dilute distributions of discs. The average flow velocity is now given by $\bar{q}=$ $-k^{e} \nabla \bar{h}$. Note that the average velocity $\bar{q}$ is referred to the bulk of the medium, while $u_{m}$ refers to the matrix domain, this means to the area outside the inclusions. The total flux is partitioned between the inclusion and the matrix as

$$
\bar{q}=(1-\chi) u_{m}+2 u_{m} \chi\langle k /(1+k)\rangle,
$$

Thus, we obtain for the characteristic matrix velocity $u_{m}$ the expression

$$
u_{m}=\frac{-k^{e} \nabla \bar{h}}{1-\chi+2 \chi\langle k /(1+k)\rangle} .
$$

The flow velocities in a unit cell of the heterogeneous medium illustrated in Figure 1 then are given by (8) with 
$u_{m}$ given by (13). Specifically, the velocity $u_{i}$ inside an inclusion is given by ( $8 \mathrm{~b})$ as

$$
u_{i}=\frac{2 u_{m} k}{1+k}
$$

The $\operatorname{PDF} p_{i}(v)$ of the $u_{i}$ can be directly related to the $\mathrm{PDF}$ of conductivity values $p_{k}(k)$ as

$$
p_{i}(v)=\frac{2 u_{m}}{\left(2 u_{m}-v\right)^{2}} p_{k}\left(\frac{v}{2 u_{m}-v}\right) .
$$

\section{STOCHASTIC PARTICLE-BASED TRANSPORT MODEL}

We focus first on particle transport in streamline coordinates, this means we consider particle movements as a function of distance $s$ along a streamline,

$$
\frac{d t(s)}{d s}=\frac{1}{v(s)}, \quad v(s)=|\mathbf{v}[\mathbf{x}(s)]|,
$$

where we set $\mathbf{x}(s) \equiv \mathbf{x}[t(s)]$. Particle motion in terms of the distance $s$ along the streamline reads as

$$
\frac{d \mathbf{x}(s)}{d s}=\mathbf{e}_{v}(s), \quad \mathbf{e}_{v}(s)=\frac{\mathbf{v}[\mathbf{x}(s)]}{v(s)} .
$$

We focus now on the particle movement along the $x$ axis of the coordinate system and choose as the coarsegraining scale the size $\ell_{0}$ of a unit cell. Thus, the particle motion can be described by

$$
x_{n+1}=x_{n}+\ell_{0}, \quad t_{n+1}=t_{n}+\tau_{n},
$$

where the transition time for a unit cell is given by

$$
\tau_{n}=\int_{s_{n}}^{s_{n}+\ell_{0}} \frac{d s^{\prime}}{v\left(s^{\prime}\right)}
$$

Due to the random nature of the permeability distribution illustrated in Figure 1, subsequent trapping times $\tau_{n}$ can be considered random and independent. Thus, the ensemble particle motion, this means particle trajectories sampled between different realizations of the random medium in Figure 1 forms a CTRW which is fully characterized by the $\operatorname{PDF} \psi(t)$ of transit times $\tau_{n}$. Before determining the particle velocities and transit time distribution in terms of the permeability distributions, we briefly recall the CTRW description of particle transport.

\section{A. Continuous Time Random Walk}

The PDF of horizontal particle positions averaged over particles and medium realizations is given by $c(x, t)=$ $\left\langle\delta\left(x-x_{n_{t}}\right)\right\rangle$, where the number of steps $n_{t}$ to reach time $t$ by the process (18) is given by $n_{t}=\max \left(n \mid t_{n} \leq t\right)$. Thus, $c(x, t)$ can be expanded as

$$
\begin{aligned}
c(x, t) & =\int_{0}^{t} d t^{\prime} R\left(x, t^{\prime}\right) \int_{t-t^{\prime}}^{\infty} d t^{\prime \prime} \psi\left(t^{\prime \prime}\right), \\
R(x, t) & =\delta(x) \delta(t)+\int_{0}^{t} d t^{\prime} \psi\left(t-t^{\prime}\right) R\left(x-\ell, t^{\prime}\right) .
\end{aligned}
$$

The first passage times (4) read now in terms of the CTRW (18) as [51]

$$
\tau\left(x_{c}\right)=t_{n_{x_{c}}}, \quad n_{x_{c}}=\min \left(n \mid x_{n} \geq x_{c}\right) .
$$

Since the spatial increment is constant equal to $\ell_{0}$, the number of steps to reach $x_{c}$ is given by $n_{x_{c}}=\left\lceil x_{c} / \ell_{0}\right\rceil$, where the upper braces denote the ceiling function. Thus, the PDF of first passage times, $f(t, r)=\langle\delta[t-\tau(r)]\rangle$ can be expanded as

$$
f\left(t, x_{c}\right)=\int_{0}^{t} d t^{\prime} f\left(t^{\prime}, x_{c}-\ell_{0}\right) \psi\left(t-t^{\prime}\right) .
$$

Note that (18)-(23) describe a CTRW for the average particle dynamics in the flow through the heterogeneous medium that is fully parameterized in terms of the distribution of hydraulic conductivity. Based on this CTRW, we also analyze the dispersion behavior in the heterogeneous porous medium. To this end, we determine the second centered moment in the average flow direction, which is defined by

$$
\sigma^{2}(t)=\left\langle\left(x_{n_{t}}-\left\langle x_{n_{t}}\right\rangle\right)^{2}\right\rangle,
$$

where $n_{t}=\sup \left(n \mid t_{n} \leq t\right)$ is the number of steps needed to reach time $t$ in the process (18). In the following, we discuss the particle velocities in the unit cell and the corresponding transit times.

\section{B. Particle Velocities and Transit Times}

We discuss here the distribution of the particle velocities entering the CTRW model outlined in the previous section and its relation to the medium properties. Furthermore, we determine the transition time distribution that corresponds to the distribution of particle velocities.

a. Velocity Distributions We simplify flow velocities in that we do not account for variability in the velocity through the matrix, and set it equal to its average $u_{m}$. According to (19), the CTRW approach outlined in the previous section requires the particle velocities $v(s)$ sampled spatially along streamlines as an input. Their PDF $p_{s}(v)$ is obtained in terms of the relative particle fluxes that pass through matrix and inclusions. The fluxes $Q_{i}$ and $Q_{m}$ through inclusions and matrix are determined at the vertical centerline of a unit cell such that

$$
Q_{i}\left(u_{i}\right)=2 u_{i}, \quad Q_{m}\left(u_{i}\right)=\left(\ell_{0}-2\right)\left(2 u_{m}-u_{i}\right)
$$


where we expressed the matrix velocity at the center line, which is given by $2 u_{m} /(1+k)$ in terms of $u_{i}$. Recall that the disc radius here is $r_{0}=1$. Thus, the conditional flux density $\mathcal{Q}\left(v \mid u_{i}\right)$ is

$$
\mathcal{Q}\left(v \mid u_{i}\right)=Q_{m}\left(u_{i}\right) \delta\left(v-u_{m}\right)+Q_{i}(v) \delta\left(v-u_{i}\right) .
$$

The global flux density $\mathcal{Q}(v)=\left\langle\mathcal{Q}\left(v \mid u_{i}\right)\right\rangle$ is obtained by averaging over the ensemble of inclusion velocities $u_{i}$ such that

$$
\mathcal{Q}(v)=\left\langle Q_{m}\left(u_{i}\right)\right\rangle \delta\left(v-u_{m}\right)+\left\langle Q_{i}\left(u_{i}\right)\right\rangle \frac{v p_{i}(v)}{\left\langle u_{i}\right\rangle} .
$$

The $\mathrm{PDF} p_{s}(v)$ of particle velocities is obtained by normalizing the flux density $\mathcal{Q}(v)$ such that

$$
p_{s}(v)=(1-\alpha) \delta\left(v-u_{m}\right)+\alpha \frac{v p_{i}(v)}{\left\langle u_{i}\right\rangle} .
$$

where we defined

$$
\alpha=\frac{\left\langle Q_{i}\left(u_{i}\right)\right\rangle}{\left\langle Q_{m}\left(u_{i}\right)\right\rangle+\left\langle Q_{i}\left(u_{i}\right)\right\rangle} .
$$

b. Transition Time Distribution The PDF of transition times consists of the distribution of transit times $\tau_{m}$ through the matrix, denoted by $\psi_{m}(t)$ and transit times $\tau_{i}$ through the inclusions, denoted by $\psi_{i}(t)$. The characteristic transit time through the matrix is given by $\tau_{0}=\ell_{0} / u_{m}$, while the minimum transition time is related to the maximum flow velocity $2 u_{m}$. Thus it is $\tau_{0} / 2$. In order to account for the flow variability in the matrix and its effect on particle dispersion, the distribution $\psi_{m}(t)$ of transition times through the matrix is modeled by a truncated exponential distribution as

$$
\psi_{m}(t)=\tau_{0}^{-1} \exp \left[-\left(t-\tau_{0} / 2\right) / \tau_{0}\right] H\left(t-\tau_{0} / 2\right)
$$

where $H(t)$ denotes the Heaviside step function. The transit times through the inclusions are estimated here in terms of an effective transition length $\ell_{i}$ of the inclusion. This effective transition length depends in general on the conductivity contrast. For a high conductivity contrast, i.e., $k \ll 1$, the velocity changes abruptly at the interface between inclusion and matrix. At the horizontal centerline, the velocity contrast at a distance $\Delta$ from the interface can be approximated by $u_{o}\left(r_{1}, 0\right) / u_{i} \approx$ $1+\Delta(1-k) / k$. Thus, for small $\langle k\rangle \ll 1$, the effective length $\ell_{i}$ is approximated by the average transition length across the disc-shaped inclusion as

$$
\ell_{i}=\frac{2}{\pi} \int_{0}^{\pi} d \varphi \cos (\varphi)=\frac{4}{\pi}
$$

Recall that the dimensionless inclusion radius is $r_{0}=1$ and that the transition length depends on the distance from the centerline. For lower conductivity contrasts between matrix and inclusion, the velocity outside the inclusion is similar to the inclusion velocity and we set $l_{i}=\ell_{0}$. The transit time through the inclusion then is given by $\tau_{i}=\ell_{i} / u_{i}$. As derived above the PDF of particle velocities in the inclusions is given by the flux weighted $v p_{i}(v) /\left\langle u_{i}\right\rangle$. Thus, we obtain for $\psi_{i}(t)$

$$
\psi_{i}(t)=\frac{\ell_{i}^{2}}{t^{3}\left\langle u_{i}\right\rangle} p_{i}\left(\ell_{i} / t\right)
$$

The transition time distribution over a unit cell is then given by

$$
\psi(t)=(1-\alpha) \psi_{m}(t)+\alpha \psi_{i}\left(t-t^{\prime}\right)
$$

The distribution of long transition time is dominated by the low end of the conductivity spectrum, at which we can set

$$
u_{i} \approx 2 u_{m} k
$$

see (8b). Thus, for $k \ll 1$, the inclusion velocity is linearly related to the inclusion conductivity so that the velocity $\mathrm{PDF}$ at small velocities can be obtained from the PDF of conductivities as

$$
p_{i}(v) \approx \frac{1}{2 u_{m}} p_{k}\left[v /\left(2 u_{m}\right)\right] .
$$

This allows to map the PDF of conductivity through (32) directly to the PDF of transition times,

$$
\psi_{i}(t) \approx \frac{\ell_{i}^{2}}{2 u_{m} t^{3}} p_{k}\left[\ell_{i} /\left(2 u_{m} t\right)\right]
$$

or in other words allows to express a transport attribute in terms of a medium property.

\section{FIRST-PASSAGE TIME DISTRIBUTIONS}

In the following, we investigate the impact of broad conductivity distributions on the long-time behavior of $f\left(t, x_{c}\right)$ and thus on the character of anomalous transport. To this end, we consider power-law distributions that behave as $p_{k}(k) \propto k^{-\gamma}$ for small conductivity values as well as broad lognormal distributions.

Within the CTRW approach derived in the previous section, first passage time distributions can be obtained straightforwardly from the Laplace transforms of (23) as $f^{*}\left(\lambda, x_{c}\right)=\psi^{*}(\lambda)^{n_{x_{c}}}$. Using the Laplace transform of $(33)$ and $(30)$

$$
f^{*}\left(\lambda, x_{c}\right)=\left[(1-\alpha) \frac{\exp \left(-\lambda \tau_{0} / 2\right)}{1+\lambda \tau_{0}}+\alpha \psi_{i}^{*}(\lambda)\right]^{n_{x_{c}}}
$$

In the limit of times much larger than $\tau_{0}$, and equivalently $\lambda \tau_{0} \ll 1$, we approximate the latter by

$$
f^{*}\left(\lambda, x_{c}\right) \approx \exp \left\{n_{x_{c}} \ln \left[1-\alpha+\alpha \psi_{i}^{*}(\lambda)\right]\right\} .
$$




\section{A. Power-Law Conductivity Distribution}

We consider the doubly truncated power-law conductivity distribution

$$
p_{k}(k)=\frac{1-\gamma}{1-k_{c}^{1-\gamma}} k_{c}^{-1}\left(\frac{k}{k_{c}}\right)^{-\gamma},
$$

for $k_{c} \leq k \leq 1$. For illustration, we consider the values $\gamma=-3 / 2$ and $\gamma=-1 / 2$. The corresponding velocity distribution is given by (15). As outlined above, we focus here on the asymptotic behavior in order to study the anomalous character of particle transport. The longtime behavior is dominated by the small particle velocities and thus through (34) to small hydraulic conductivities. Thus (39) implies here that the velocity distribution behaves as $p_{i}(v) \propto\left(v / u_{m}\right)^{-\gamma}$, see (35), and from (36) that the transition time PDF scales as

$$
\psi_{i}(t) \propto\left(t / \tau_{a}\right)^{\gamma-3}
$$

for $t \ll \tau_{c}$, where we defined the cut-off time scale $\tau_{c}=$ $\ell_{i} /\left(2 u_{m} k_{c}\right)$, which corresponds to the lower conductivity cut-off $k_{c}$. Furthermore, we define the time scale $\tau_{a}=$ $\ell_{i} /\left(2 u_{m}\right)$, which corresponds to the upper conductivity cut-off of 1 . We consider here $0<\gamma<2$. For $1<\gamma<2$, the Laplace transform of the transition time distribution can be expanded as [22]

$$
\psi_{i}^{*}(\lambda)=1-a_{\gamma}\left(\lambda \tau_{a}\right)^{2-\gamma}
$$

for $\tau_{c}^{-1} \ll \lambda \ll \tau_{a}^{-1}$, while for $0<\gamma<1$, we obtain [22]

$$
\psi_{i}^{*}(\lambda)=1-\lambda \tau_{a v}+b_{\gamma}\left(\lambda \tau_{a}\right)^{2-\gamma},
$$

where we defined the mean transition time $\tau_{a v}=\ell_{i} /\left\langle u_{i}\right\rangle$.

Inserting the expansion (41) into (38), we obtain

$$
f^{*}\left(\lambda, x_{c}\right) \approx \exp \left[-n_{x_{c}} \alpha a_{\gamma}\left(\lambda \tau_{a}\right)^{2-\gamma}\right],
$$

which is a skewed Levy-stable density. Its inverse Laplace transform has the scaling form $f\left(t, x_{c}\right)=$ $\left(\alpha a_{\gamma} x_{c}\right)^{-1 /(2-\gamma)} f_{0}\left[t /\left(\alpha a_{\gamma} x_{c}\right)^{1 /(2-\gamma)}\right]$. The scaling function $f_{0}(t)$ has the Laplace transform $f_{0}^{*}(\lambda)=$ $\exp \left[-\left(\lambda \tau_{a}\right)^{2-\gamma}\right]$. The long time behavior of $f\left(t, x_{c}\right)$ is given by $f\left(t, x_{c}\right) \propto t^{\gamma-3}$. Inserting the expansion (42) into (38), we obtain

$$
f^{*}\left(\lambda, x_{c}\right) \approx \exp \left\{-n_{x_{c}} \alpha\left[\lambda \tau_{a v}-b_{\gamma}\left(\lambda \tau_{a}\right)^{2-\gamma}\right]\right\},
$$

Thus, the long-time behavior for the $f\left(t, x_{c}\right)$ is also given by $f\left(t, x_{c}\right) \propto t^{\gamma-3}$.

Figure 2 shows the first-passage time distributions obtained from direct numerical simulations of particle transport in the flow through heterogeneous conductivity fields characterized by the point distribution (39) for $\gamma=3 / 2$ and $\gamma=1 / 2$. The simulation data are compared to the predictions of the corresponding CTRW model described in Section III. The late time power-law scaling is indicated by the dashed lines. The CTRW model provides an accurate prediction of the late time scaling of the
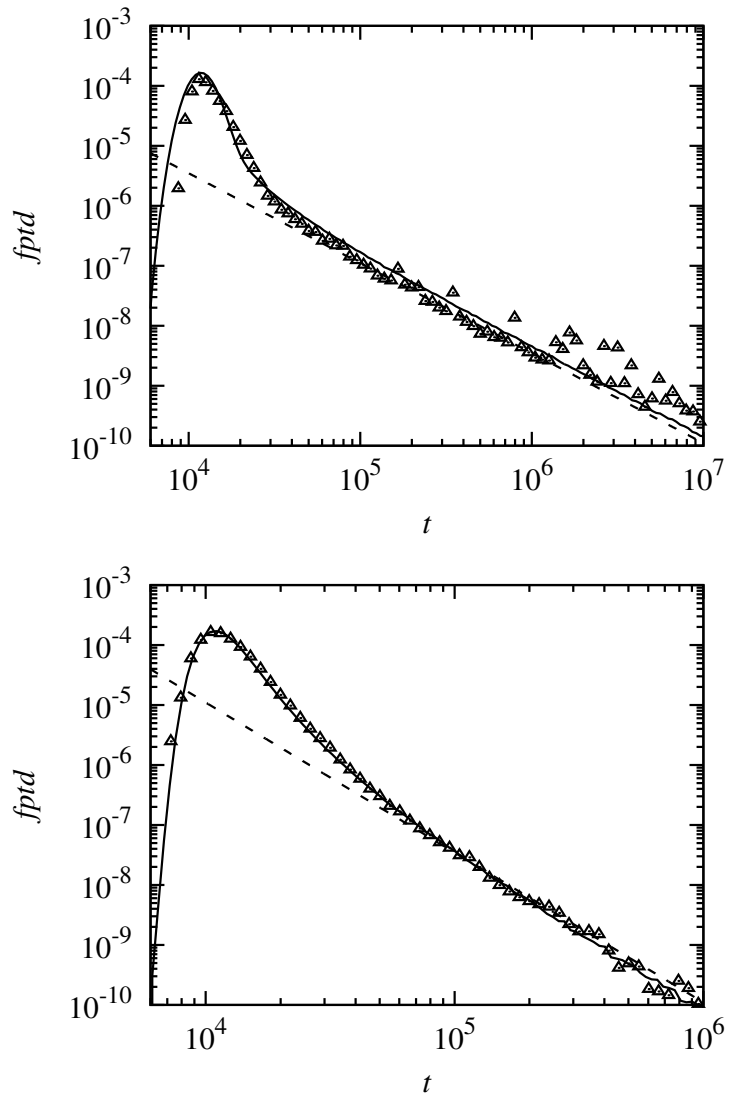

FIG. 2. First passage time distributions obtained from (triangles) direct numerical simulation of particle transport in the heterogeneous porous medium and (solid lines) the prediction of the CTRW model, for the power-law $k$-distribution (39) with $k_{c}=10^{-5}$ and (top panel) $\gamma=3 / 2$ or respectively (bottom panel) $\gamma=1 / 2$. The dashed lines indicate the power-law behavior $\propto t^{\gamma-3}$ expected in the intermediate time regime. The CTRW simulation parameters are given in Appendix A 2. The direct numerical simulations are described in Appendix A 1.

first passage time distributions. The late time scaling is directly related to the behavior of $p_{k}(k)$ for $k \ll 1$. The peak behavior is captured satisfactorily by the truncated exponential distribution of particle transit times in the matrix.

\section{B. Lognormal Conductivity Distribution}

We consider now the truncated lognormal distribution of conductivities

$$
p_{k}(k)=\sqrt{\frac{2}{\pi \sigma^{2}}} \frac{\exp \left[-\frac{(\ln k-\mu)^{2}}{2 \sigma^{2}}\right]}{k \operatorname{erfc}\left(\mu / \sqrt{2 \sigma^{2}}\right)}
$$

for $0<k<1$. The transition time distribution $\psi_{i}(t)$ is given by (32) in terms of the velocity distribution through the inclusions. As pointed out above, for $k \ll 1$ we may 
set $u_{i} \approx 2 u_{m} k$, see (8b). This allows to relate the velocity $\mathrm{PDF} p_{i}(v)$ to the conductivity PDF according to (35) and the transition time $\mathrm{PDF} \psi_{i}(t)$ to $p_{k}(k)$ through (36). It follows that the transition time $\operatorname{PDF} \psi_{i}(\tau)$ has itself the form of a truncated lognormal distribution. Thus, unlike in the previous section, where the CTRW predicts a power-law behavior of the first passage time distribution as a consequence of the generalized central limit theorem here this is not the case.

Figure 3 shows first-passage time distributions obtained from direct numerical simulations in conductivity fields characterized by the lognormal conductivity distribution (45) for $\sigma^{2}=11.4$ and $\mu=-9.23$ and $\mu=2.3$. The CTRW model developed in Section III provides a good prediction of the tailing behavior and captures the peak behavior satisfactorily.

Edery et al. [26] proposed to fit a power-law to the low- $k$ end of $p_{k}(k)$ corresponding to the time regime, for which a prediction is desired. The resulting power-law approximation may then be used to make an approximation on the tailing behavior of the first-passage time distribution. Note that (45) may be expanded into a power-law around any $k_{0}<1$ as

$$
p_{k}(k) \propto k^{-\gamma}, \quad \gamma=\frac{\sigma^{2}-\mu+\ln k_{0}}{\sigma^{2}} .
$$

This can be readily seen by expanding $\ln p_{k}(k)$ around $\ln k_{0}$ up to linear order. For small $k$, the first-passage time $\tau_{i}$ through the inclusions are related to $k$ as $\tau_{i}=$ $\ell_{i} /\left(2 u_{m} k\right)$. This means first passage times of the order of a $t_{0}$ correspond to

$$
k_{0} \sim \ell_{i} /\left(2 u_{m} t_{0}\right) .
$$

Thus, (46) together with (47) may be used as a rough approximation for the tail scaling around a first-passage time $t_{0}$. The dashed lines in Figure 3 indicate the scaling approximation around $t_{0}=10^{5}$. For $\sigma^{2}=11.4$ and $\mu=-9.23$, we obtain from (46) that $\gamma=1.1$. For $\sigma^{2}=11.4$ and $\mu=-2.3$ we obtain $\gamma=0.4$. These estimates of $\gamma$ provide a good approximation to the tailing behavior around $t_{0}=10^{5}$. Note, however, that unlike in the previous section, here, the first passage time distributions do not exhibit power-law tails because the first passage times, here are obtained through the summation of lognormally distributed random variables.

\section{DISPERSION}

In the previous section, we have demonstrated the predictive capabilities of the CTRW model derived in Section III for global particle transport in a heterogeneous medium characterized by a broad distribution of hydraulic conductivities. In this section, we use this model in order to analyze particle dispersion and its controls in terms of the distribution of hydraulic conductivities.
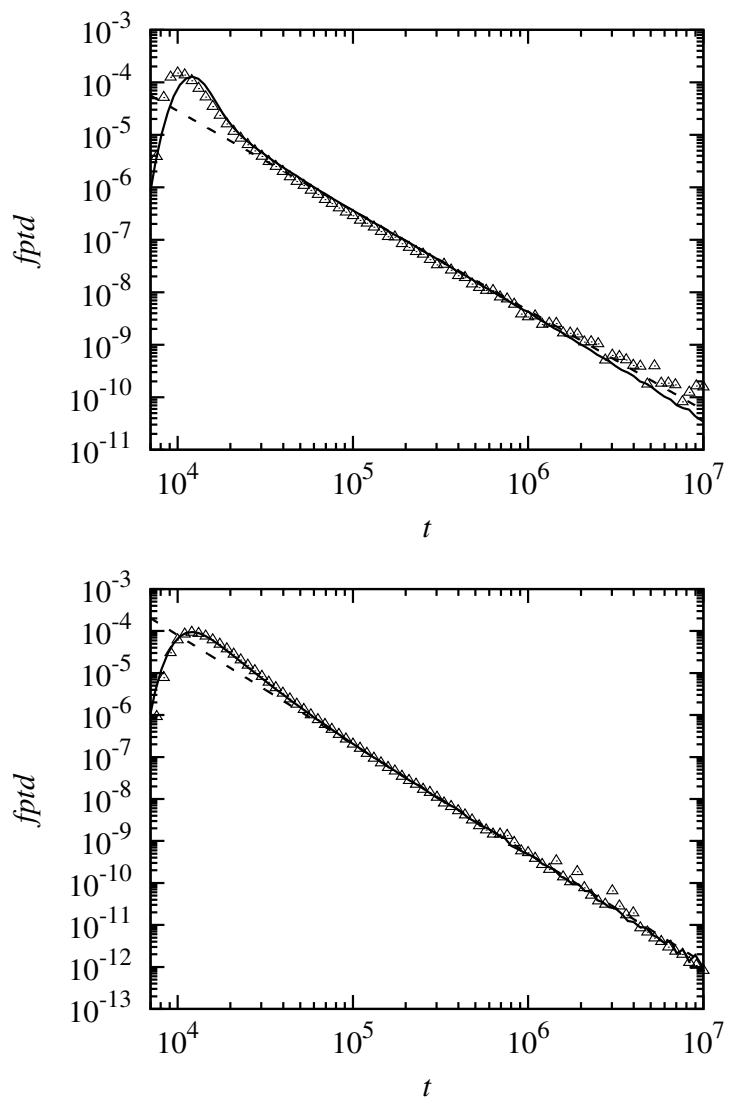

FIG. 3. First passage time distributions obtained from (triangles) direct numerical simulation of particle transport in the heterogeneous porous medium, see Appendix A 1 and (solid lines) the prediction of the CTRW model, for the lognormal $k$-distribution (45) with $\sigma^{2}=11.4$ and (top panel) $\mu=-9.23$ or respectively (bottom panel) $\mu=-2.3$. The CTRW simulation parameters are given in Appendix A 2. The dashed lines indicate the approximate power-law behavior obtained from (46) and (47).

Dispersion is measured in terms of the centered mean square displacement

$$
\kappa(t)=m_{2}(t)-m_{1}(t)^{2} .
$$

The first and second displacement moments are defined in the CTRW framework as

$$
m_{1}(t)=\left\langle x_{n_{t}}\right\rangle, \quad m_{2}(t)=\left\langle x_{n_{t}}^{2}\right\rangle,
$$

where $n_{t}=\sup \left(n \mid t_{n} \leq t\right)$. We obtain explicit Laplace space expressions for $m_{1}^{*}(\lambda)$ and $m_{2}^{*}(\lambda)$ in terms of the transition time distribution $\psi^{*}(\lambda)[22,52]$

$$
\begin{aligned}
& m_{1}^{*}(\lambda)=\frac{\ell_{0}}{\lambda^{2}} \frac{\lambda \psi^{*}(\lambda)}{1-\psi^{*}(\lambda)} \\
& m_{2}^{*}(\lambda)=\frac{\ell_{0}^{2}}{\lambda^{2}} \frac{\lambda \psi^{*}(\lambda)}{1-\psi^{*}(\lambda)}+2 \frac{\ell_{0}^{2}}{\lambda^{3}} \frac{\lambda^{2} \psi^{*}(\lambda)}{\left[1-\psi^{*}(\lambda)\right]^{2}} .
\end{aligned}
$$

The Laplace transform of the transition time distribution 
here is given by

$$
\psi^{*}(\lambda)=(1-\alpha) \psi_{m}^{*}(\lambda)+\alpha \psi_{i}^{*}(\lambda) .
$$

For transition time distributions characterized by finite mean and mean square transition times, $\kappa(t)$ increases linearly with time, $\kappa(t)=2 D^{e} t$. The effective dispersion coefficient is given in terms of the first and second moments of $\psi(t)$ as [22]

$$
D^{e}=\frac{\ell_{0}^{2}}{2} \frac{\left\langle\tau^{2}\right\rangle-\langle\tau\rangle^{2}}{\langle\tau\rangle^{3}} .
$$

For the composite transition time distribution (33) the $i$-th moment $\left\langle\tau^{i}\right\rangle$ of the transition time is given by

$$
\left\langle\tau^{i}\right\rangle=(1-\alpha)\left\langle\tau_{m}^{i}\right\rangle+\alpha\left\langle\tau_{i}^{i}\right\rangle .
$$

For the first and second moments of the mobile transition time, we obtain from (30)

$$
\left\langle\tau_{m}\right\rangle=\tau_{0}+\tau_{0} / 2, \quad\left\langle\tau_{m}^{2}\right\rangle=\tau_{0} / 2^{2}+2 \tau_{0}\left(\tau_{0}+\tau_{0} / 2\right) .
$$

For the truncated power-law distribution (39), the transition time distribution $\psi_{i}(t)$ can be approximated by the truncated power-law

$$
\psi_{i}(t)=\frac{2-\gamma}{\tau_{a}} \frac{\left(t / \tau_{a}\right)^{\gamma-3}}{1-\left(\tau_{c} / \tau_{a}\right)^{\gamma-2}}
$$

for $\tau_{a}<t<\tau_{c}$. The cut-off time $\tau_{c}$ is related to the smallest conductivity value as $\tau_{c}=\ell_{i} /\left(2 u_{m} k_{c}\right)$.

\section{A. Intermediate Time Regime}

We first consider dispersion in the intermediate time regime $\tau_{a} \ll t \ll \tau_{c}$. It behaves as $\psi(t) \propto t^{\gamma-3}$. In this time regime, dispersion is anomalous and characterized by the following scalings. For $1<\gamma<2$, one obtains [22]

$$
\kappa(t) \propto t^{4-2 \gamma} .
$$

For $0<\gamma<1$, one finds

$$
\kappa(t) \propto t^{1+\gamma} .
$$

The second centered moment of the particle distribution increases superdiffusively.

\section{B. Long Time Regime}

Now we investigate the dependence of the effective dispersion coefficient (53) on the cut-off time scale $\tau_{c}$ and equivalently on the minimum conductivity scale $k_{c}$. The first and second moments of the transition time distribution $\psi_{i}(t)$ are obtained from (56) as

$$
\begin{aligned}
\left\langle\tau_{i}\right\rangle & =\tau_{a} \frac{2-\gamma}{1-\gamma} \frac{1-\left(\tau_{c} / \tau_{a}\right)^{\gamma-1}}{1-\left(\tau_{c} / \tau_{a}\right)^{\gamma-2}} \\
\left\langle\tau_{i}^{2}\right\rangle & =\tau_{a}^{2} \frac{2-\gamma}{\gamma} \frac{\left(\tau_{c} / \tau_{a}\right)^{\gamma}-1}{1-\left(\tau_{c} / \tau_{a}\right)^{\gamma-2}} .
\end{aligned}
$$

We furthermore note that $\alpha \propto\left\langle u_{i}\right\rangle \approx 2 u_{m}\langle k\rangle$, where the mean conductivity over the inclusions is obtained from (39) as

$$
\langle k\rangle=\frac{1-\gamma}{2-\gamma} \frac{1-k_{c}^{2-\gamma}}{1-k_{c}^{1-\gamma}}
$$

This means, for $0<\gamma<1$, the mean conductivity converges to the finite value $\langle k\rangle=(1-\gamma) /(2-\gamma)$ in the limit $k_{c} \rightarrow 0$, while for $1<\gamma<2$ it goes toward 0 as $\langle k\rangle \propto k_{c}^{\gamma-1}$. In the following, we quantify the behavior of the dispersion coefficient (53) in the limit of $\tau_{c} \gg \tau_{a}$.

a. $0<\gamma<1$ We first consider the case $0<\gamma<1$. In this case, we obtain for the transition time moments in leading order

$$
\begin{aligned}
\left\langle\tau_{i}\right\rangle & =\tau_{a} \frac{2-\gamma}{1-\gamma} . \\
\left\langle\tau_{i}^{2}\right\rangle & =\tau_{a}^{2} \frac{2-\gamma}{\gamma}\left(\tau_{c} / \tau_{a}\right)^{\gamma} .
\end{aligned}
$$

For the mean conductivity, we obtain

$$
\langle k\rangle=\frac{1-\gamma}{2-\gamma} .
$$

This means, both $\alpha$ and $\langle\tau\rangle$ are constant in the limit of larger $\tau_{c}$. Thus, dispersion coefficient (53) is dominated by $\left\langle\tau_{i}^{2}\right\rangle$, which increases as $\tau_{c}^{\gamma}$. Thus, in leading order, we can write

$$
D^{e}=\frac{\ell_{0}^{2}}{2} \frac{2-\gamma}{\gamma} \alpha \frac{\tau_{a}^{2}}{\langle\tau\rangle^{2}}\left(\tau_{c} / \tau_{a}\right)^{\gamma} \propto k_{c}^{-\gamma}
$$

This means, the effective dispersion coefficient increases monotonically with increasing conductivity contrast between the inclusions.

b. $1<\gamma<2$ Unlike in the case $\gamma<1$, here $k_{c} \rightarrow 0$ is a singular limit for the conductivity distribution because the normalizability of $p_{k}(k)$ depends on the finiteness of $k_{c}$. We obtain for the mean conductivity $\langle k\rangle$ in the limit $k_{c} \ll 1$ the leading behavior

$$
\langle k\rangle=\frac{\gamma-1}{2-\gamma} k_{c}^{\gamma-1} .
$$

This means, the mean inclusion conductivity, and therefore the mean velocity through the inclusions go to 0 in the limit $k_{c} \rightarrow 0$. In this limit, the inclusions are on average impermeable. Based on (65), we may now write $\alpha$ as

$$
\alpha=\hat{\alpha}\left(\tau_{c} / \tau_{a}\right)^{1-\gamma}, \quad \hat{\alpha}=\alpha\left(\tau_{c} / \tau_{a}\right)^{\gamma-1},
$$

where $\hat{\alpha} \rightarrow$ constant in the limit $k_{c} \rightarrow 0$. For the mean and mean squared transition times through the inclusions, we obtain from (59) in leading order for $\tau_{c} \gg \tau_{a}$

$$
\begin{aligned}
\left\langle\tau_{i}\right\rangle & =\tau_{a} \frac{2-\gamma}{\gamma-1}\left(\tau_{c} / \tau_{a}\right)^{\gamma-1} \\
\left\langle\tau_{i}^{2}\right\rangle & =\tau_{a}^{2} \frac{2-\gamma}{\gamma}\left(\tau_{c} / \tau_{a}\right)^{\gamma} .
\end{aligned}
$$


Thus, we obtain in leading order for the effective dispersion coefficient

$$
D^{e}=\frac{\ell_{0}^{2}}{2} \frac{2-\gamma}{\gamma} \frac{\tau_{a}^{2}}{\langle\tau\rangle^{3}} \hat{\alpha} \tau_{c} / \tau_{a} \propto k_{c}^{-1} .
$$

This means, it increases linearly with the cut-off time scale $\tau_{c}$ and is inversely proportional to the minimum conductivity $k_{c}$.

Note that the scaling behaviors (64) and (69) are universal for any conductivity distribution that shows a (truncated) power-law behavior for $k_{c}<k \ll 1$ as $p_{k}(k) \propto k^{-\gamma}$. Note also that for any conductivity distribution that has the scaling form

$$
p_{k}(k)=\frac{1}{k_{c}} f_{k}\left(k / k_{c}\right),
$$

with $f_{k}(k)$ a scaling function that is normalized to 1 , one finds that $\alpha \propto k_{c},\left\langle\tau_{i}\right\rangle \propto k_{c}^{-1}$ and $\left\langle\tau_{i}^{2}\right\rangle \propto k_{c}^{-1}$. Thus in the limit of $k_{c} \rightarrow 0$ one finds here that $D_{e} \propto k_{c}^{-1}$, see also Ref. [40].

\section{SUMMARY AND CONCLUSIONS}

We investigated the mechanisms of anomalous dispersion in the flow through heterogeneous porous media. To this end, we considered a medium composed of equally sized inclusions embedded in a porous matrix. The hydraulic conductivities of the inclusions are broadly distributed with tails toward small values. Such media can be seen as idealizations of highly heterogeneous porous media that have a characteristic correlation scale. The conductivities in the inclusions is mapped onto the flow velocities through an analytical expression. The background velocity is related to the effective conductivity of the medium, which is obtained from the Maxwell formula.

Based on these results we formulate the purely advective movement of solute particles in terms of travel distance along streamlines, which renders the equations of motion a CTRW, in which the transition length is fixed through the characteristic heterogeneity length scale and the transition time is related to the particle velocities. We define unit cells as a domain that contains a single inclusion. When crossing a unit cell particles can bypass the inclusion or pass through. The probabilities for the respective path are given by the fluxes though matrix and inclusions. Thus the PDF of particle velocities is obtained from the PDF of flow velocities through flux weighting. This provides a direct link between the medium properties through the explicit relation between flow velocities and hydraulic conductivity.

The derived CTRW model is then used to predict first passage time distributions obtained from particle tracking simulations in the flow through heterogeneous media characterized by different heavy-tailed distributions of hydraulic conductivities. Specifically, we consider truncated power-law behaviors at small conductivities and broad truncated lognormal distributions. The power-law in the conductivity is directly mapped onto a power-law of transition times, which predicts the power-law behavior observed in the direct numerical simulations as a consequence of the generalized central limit theorem. Also for the lognormal conductivity distributions, we observe broad distributions of first-passage times. Here however, they are not power-laws because they result from an addition of lognormally distributed random variables. Nevertheless, for very broad conductivity distributions, the lognormal PDF may be approximated by a power-law with an exponent determined by the mean and variance of the log-hydraulic conductivity. This may be used to describe the tailing behavior in certain time ranges based on the expressions derived for power-law distributions. The heavy tails are eventually tempered at time scales that correspond to the smallest hydraulic conductivity values.

Based on the derived CTRW model, we investigate the dispersion behavior in terms of the second centered moments of the particle distribution, or centered mean square displacement. The CTRW predicts anomalous dispersion characterized by non-linear evolutions of the centered mean square displacement. Specifically, for the truncated power-law distribution of hydraulic conductivity CTRW predicts a power-law evolution. At asymptotically long times, much larger than the cut-off time scale, dispersion becomes normal as a consequence of the central limit theorem. The corresponding dispersion coefficients are quantified in terms of the conductivity distributions.

The medium under consideration is $d=2$ dimensional. The developed CTRW model, however, can be straightforwardly generalized to $d=3$ dimensional media based on similar analytical expressions for the flow velocity inside the inclusions [41]. Furthermore, the present study considers purely advective transport. Diffusion into lowconductivity inclusions would introduce an additional cut-off scale for the transition time distribution if the characteristic diffusion time over the inclusion is smaller than the largest advection time scale. These behaviors are subject of ongoing research.

In conclusion, the derived CTRW model provides a predictive description of transport through highly heterogeneous media based on the distribution of hydraulic conductivity and characteristic heterogeneity length scales. The concrete heterogeneity model is a caricature of highly heterogeneous porous media characterized by finite correlation scales. Thus, the presented results shed light on the fundamental mechanisms of anomalous dispersion and its relations with the medium and flow properties.

\section{Acknowledgments}

The authors gratefully acknowledge financial support by the Swiss National Science Foundation (SNF) through 
grant 200021_132304 and by Nagra, Wettingen. MD acknowledges the support of the European Research Council (ERC) through the project MHetScale (617511).

\section{Appendix A: Numerical Simulations}

In the following, we give some details on the numerical simulations. First, we describe the setup of the direct numerical simulations of flow and particle transport in the conductivity fields characterized by low-conductivity inclusions embedded in a higher conductive matrix. Then, we give the details for the particle tracking simulation that implement the CTRW model developed in Section III.

\section{Flow and Transport Simulations}

We consider a regular field size of 512 by 512 cells, consisting of a highly conductive matrix with $k_{0}=1$ and a set of circular low conductivity inclusions with radii $r_{0}=$ 15 and centers in $\left(x_{i}, y_{j}\right): y_{j}=32 j+s_{i}, x_{i}=32 i+16$, where $s_{i}$ is a random shift (Figure 1). Hydraulic conductivities $k$ inside inclusions are independent identically distributed random variables with the PDF $p_{k}(k)$.

Flow is driven by a constant hydraulic head gradient between the inlet boundary at $x=0$ and the outlet boundary at $x=512,|\nabla \bar{h}|=\Delta h / 512=[h(512, y)-$ $h(0, y)] / 512=-0.1$. The flow field is solved numerically using a finite difference scheme with a unit discretization $\Delta x=\Delta y=1$ [53]. This means 30 cells per inclusion diameter. Such a fine discretization is required due to the high conductivity contrast between inclusions and matrix.

Transport is solved by particle tracking based on the advection equation (3) using the scheme of Pollock [54]. The Pollock algorithm interpolates the flow velocity within a finite difference cell bi-linearly to guarantee that the divergence of the flow velocity is 0 [55]. This interpolation is necessary because the finite difference method gives only the flow velocities perpendicular to the cell facies. The trajectory within the cell is then determined analytically by integration of the advection equation (3), which gives the Pollock integral. The particle tracking simulations use $10^{5}$ particles, which are injected proportional to the flux at the surface at $x=30$. The observation plain is the right boundary of the domain at $x=512$. The first passage times are determined according to (4) through an average over the $10^{5}$ particles in single realizations and between $10^{3}$ realization for each random field under consideration.

\section{CTRW Simulations}

The CTRW model is based on the stochastic recursion relation (18). The first passage times are determined according to (23). The transition time distribution $\psi(t)$ is given by (33). It requires the parameters $\alpha$ given by (29), the matrix velocity $u_{m}$ given by (13), which depends on the effective conductivity $k_{e}$ given by (10), the average inclusion velocity $\left\langle u_{i}\right\rangle$ and the effective length $\ell_{i}$. The volume fraction of the inclusion is $\chi=0.69$. The flux weighted velocity distributions $v_{i} p_{i}(v) /\left\langle v_{i}\right\rangle$ are generated by the rejection method. To this end, we consider the corresponding flux-weighted conductivity distribution

$$
\hat{p}_{k}(k)=\frac{k}{1+k} p_{k}(k) /\langle k /(1+k)\rangle
$$

and compare it to $c p_{k}(k)$, where $c$ is chosen such that that $c p_{k}(k) \geq \hat{p}_{k}(k)$. The CTRW simulations reported in Figures 2 and 3 use $10^{7}$ particles.

\section{a. Power-law Conductivity Distribution}

For the power-law conductivity distribution (39) we obtain the following parameters. For $\gamma=3 / 2$, the effective conductivity is $k^{e}=0.186$, the matrix velocity is $u_{m}=0.059$, the mean inclusion velocity is $\left\langle u_{i}\right\rangle=0.00036$ the trapping frequency is $\alpha=0.036$. The effective immobile length is here set to $l_{i}=4 r / \pi=19$ because the mean conductivity in the inclusion is $\langle k\rangle=0.0032 \ll 1$.

For $\gamma=1 / 2$, the effective conductivity is $k^{e}=0.436$, the matrix velocity is $u_{m}=0.072$, the mean inclusion velocity is $\left\langle u_{i}\right\rangle=0.031$ the trapping frequency is $\alpha=0.8$. The effective immobile length is here set to $l_{i}=32$ because the mean conductivity in the inclusion is $\langle k\rangle=0.334$. Regarding the reasoning for the choice of the effective transition length $l_{i}$, see also the discussion in Section III B.

\section{b. Log-normal Conductivity Distribution}

For the lognormal conductivity distribution (45) we obtain the following parameters. For $\mu=-9.23$ and $\sigma^{2}=$ 11.4, the effective conductivity is $k^{e}=0.19$, the matrix velocity is $u_{m}=0.059$, the mean inclusion velocity is $\left\langle u_{i}\right\rangle=0.00073$ the trapping frequency is $\alpha=0.085$. The effective immobile length is here set to $l_{i}=4 r_{0} / \pi=19$ because the mean conductivity in the inclusion is $\langle k\rangle=$ $0.0077 \ll 1$.

For $\mu=-2.3$ and $\sigma^{2}=11.4$, the effective conductivity is $k^{e}=0.29$, the matrix velocity is $u_{m}=0.064$, the mean inclusion velocity is $\left\langle u_{i}\right\rangle=0.012$ the trapping frequency is $\alpha=0.62$. The effective immobile length is here set to $l_{i}=23$ because the mean conductivity in the inclusion is $\langle k\rangle=0.14$, this means the contrast between the matrix conductivity and average inclusion conductivity is relatively low, see also the discussion in Section III B. 
[1] Y. Hatano and N. Hatano, "Dispersive transport of ions in column experiments: An explanation of long-tailed profiles," Water Resour Res 34 (5), 1027-1033 (1998).

[2] B. Berkowitz, A. Cortis, M. Dentz, and H. Scher, "Modeling non-fickian transport in geological formations as a continuous time random walk," Rev. Geophys. 44 (2006), 10.1029/2005RG000178.

[3] S. P. Neuman and D. M. Tartakovsky, "Perspective on theories of anomalous transport in heterogeneous media," Adv. Water Resour. 32, 670-680 (2008).

[4] P. K. Kang, T. Le Borgne, M. Dentz, O. Bour, and R. Juanes, "Impact of velocity correlation and distribution on transport in fractured media: field evidence and theoretical model," Water Resour. Res. 51, 940-959 (2015).

[5] Aldo Fiori and Matthew W. Becker, "Power law breakthrough curve tailing in a fracture: The role of advection," J. Hydrol. 525, 706-710 (2015).

[6] A Bellin, A Rinaldo, WJP Bosma, SEATM Vanderzee, and Y Rubin, "Linear equilibrium adsorbing solute transport in pphysically and chemically heterogeneous porous formations. 1. analytical solutions," Water Resour. Res. 29, 4019-4030 (1993).

[7] D. Bolster and M. Dentz, "Anomalous dispersion in chemically heterogeneous media induced by long-range disorder correlation," J. Fluid Mech. 695, 366-389 (2012).

[8] I. Neretnieks and A. Rasmuson, "An approach to modeling radionuclide migration in a medium with strongly varying velocity and block sizes along the flow path," Water Resour. Res. 20, 1823-1836 (1984).

[9] A. Comolli, J. Hidalgo, C. Moussey, and M. Dentz, "Non-fickian transport under heterogeneous advection and mobile- immobile mass transfer," Transp. Porous Media 113 (2016), 10.1007/s11242-016-0727-6.

[10] M. W. Becker and A. Shapiro, "Tracer transport in fractured crystalline rock: Evidence of nondiffusive breakthrough tailing," Water Resour. Res. 36, 1677-1686 (2000).

[11] G. Di Donato, E. Obi, and M. J. Blunt, "Anomalous transport in heterogeneous media demonstrated by streamline-based simulation," Geophys. Res. Lett. 30, 1608 (2003).

[12] MJ Ronayne and SM Gorelick, "Effective permeability of porous media containing branching channel networks," Phys Rev. E 73 (2006), 10.1103/PhysRevE.73.026305.

[13] A. R. Tyukhova, W. Kinzelbach, and M. Willmann, "Delineation of connectivity structures in 2-d heterogeneous hydraulic conductivity fields," Water Resour. Res. 51, 5846-5854 (2015).

[14] A.R. Tyukhova and M. Willmann, "Connectivity metrics based on the path of smallest resistance," Advances in Water Resources 88, 14-20 (2016).

[15] E.W. Montroll and G.H. Weiss, "Random walks on lattices. ii," J. Math. Phys. 6, 167 (1965).

[16] H. Scher and M. Lax, "Stochastic transport in a disordered solid. I. Theory," Phys. Rev. B 7, 4491-4502 (1973).

[17] M. F. Shlesinger, B. J. West, and J. Klafter, "Lévy dynamics of enhanced diffusion: Application to turbulence," Phys. Rev. Lett. 58, 1100-1103 (1987).
[18] Ralf Metzler and Joseph Klafter, "The random walk's guide to anomalous diffusion: a fractional dynamics approach," Phys. Rep. 399, 1-77 (2000).

[19] J. Klafter and I. Sokolov, "Anomalous diffusion spreads its wings," Phys. World 18, 29-32 (2005).

[20] Simon Thalabard, Giorgio Krstulovic, and Jérémie Bec, "Turbulent pair dispersion as a continuous-time random walk," J. Fluid Mech. 755, R4 (2014).

[21] B. Berkowitz and H. Scher, "Anomalous transport in random fracture networks," Phys. Rev. Lett. 79, 4038-4041 (1997).

[22] M. Dentz, A. Cortis, H. Scher, and B. Berkowitz, "Time behavior of solute transport in heterogeneous media: transition from anomalous to normal transport," Adv. Water Resour. 27, 155-173 (2004).

[23] Tanguy Le Borgne, Marco Dentz, and Jesus Carrera, "Lagrangian statistical model for transport in highly heterogeneous velocity fields," Phys. Rev. Lett. 101 (2008), 10.1103/PhysRevLett.101.090601.

[24] B Berkowitz and H Scher, "Theory of anomalous chemical transport in random fracture networks," Phys. Rev. E 57, 5858-5869 (1998).

[25] P. K. Kang, M. Dentz, T. Le Borgne, and R. Juanes, "Spatial markov model of anomalous transport through random lattice networks," Phys. Rev. Lett. 107, 180602 (2011).

[26] Y. Edery, A. Guadagnini, H. Scher, and B. Berkowitz, "Origins of anomalous transport in heterogeneous media: Structural and dynamic controls," Water Resources Research 50, 1490-1505 (2014), cited By 17.

[27] R. Haggerty and S.M. Gorelick, "Multiple-rate masstransfer for modeling diffusion and surface-reactions in media with pore-scale heterogeneity," Water Resour. Res. 31, 2383-2400 (1995).

[28] J. Carrera, X. Sánchez-Vila, I. Benet, A. Medina, G. Galarza, and J. Guimerà, "On matrix diffusion: formulations, solution methods, and qualitative effects," Hydrogeology Journal 6, 178-190 (1998).

[29] R. Haggerty, S. A. McKenna, and L. C. Meigs, "On the late time behavior of tracer test breakthrough curves," Water Resour. Res. 36, 3467-3479 (2000).

[30] M. Dentz and B. Berkowitz, "Transport behavior of a passive solute in continuous time random walks and multirate mass transfer," Water Resour. Res. 39, 1111 (2003).

[31] R. Schumer, D. A. Benson, M. M. Meerschaert, and B. Baeumer, "Fractal mobile/immobile solute transport," Water Resour Res 39(10), 1296 (2003).

[32] D. A. Benson and M. M. Meerschaert, "A simple and efficient random walk solution of multi-rate mobile/immobile mass transport equations," Adv. Water Resour. 32, 532-539 (2009).

[33] M. Willmann, J. Carrera, and X. Sànchez-Vila, "Transport upscaling in heterogeneous aquifers: What physical parameters control memory functions?" Water Resour. Res. 44 (2008), 10.1029/2007WR006531.

[34] Y. Zhang, C. T. Green, and G. E. Fogg, "The impact of medium architecture of alluvial settings on non-fickian transport," Adv. Water Resour. 54, 78-99 (2013).

[35] Alina R. Tyukhova and Matthias Willmann, "Conservative transport upscaling based on information of connec- 
tivity," Water Resources Research 52, n/a-n/a (2016).

[36] J. P. Bouchaud and A. Georges, "Anomalous diffusion in disordered media: Statistical mechanisms, models and physical applications," Phys. Rep. 195, 127-293 (1990).

[37] M. Dentz and A. Castro, "Effective transport dynamics in porous media with heterogeneous retardation properties," Geophys. Res. Lett. 36, L03403 (2009).

[38] M. Dentz and D. Bolster, "Distribution- versus correlation-induced anomalous transport in quenched random velocity fields," Phys. Rev. Lett. 105, 244301 (2010).

[39] P. K. Kang, Dentz. M., and R. Juanes, "Predictability of anomalous transport on lattice networks with quenched disorder," Phys. Rev. E 83, 030101(R) (2011).

[40] I. Eames and J. W. M. Bush, "Longitudinal dispersion by bodies fixed in potential flow." Proc. R. Soc. Lond. A 455, 3665-3686 (1999).

[41] A. Fiori, I. Jankovic, and G. Dagan, "Modeling flow and transport in highly heterogeneous three-dimensional aquifers: Ergodicity, gaussianity, and anomalous behavior - 2. approximate semianalytical solution," Water. Resour. Res. 42, W06D13 (2006).

[42] A. Fiori, I. Janković, Dagan G., and V. Cvetković, "Ergodic transport through aquifers of non-gaussian log conductivity distribution and occurrence of anomalous behavior," Water Resour. Res. 43, W09407 (2007).

[43] A. Fiori, G. Dagan, I. Jankovic, and A. Zarlenga, "The plume spreading in the made transport experiment: Could it be predicted by stochastic models?" Water Resour. Res. 49, 2497-2507 (2013).

[44] V. Cvetkovic, A. Fiori, and G. Dagan, "Solute transport in aquifers of arbitrary variability: A time-domain ran- dom walk formulation," Water Resources Research 50, 5759-5773 (2014), cited By 6.

[45] J. Bear, Dynamics of fluids in porous media (American Elsevier, New York, 1972).

[46] S. W. Wheatcraft and F. Winterberg., "Steady state flow passing through a cylinder a permeability different from the surrounding medium," Water Resour. Res. 21, 19231929 (1985).

[47] P. Renard and G. de Marsily, "Calculating equivalent permeability: A review," Adv. Water Resour. 20, 253278 (1997).

[48] X. Sanchez-Vila, A. Guadagnini, and J. Carrera, "Representative hydraulic conductivities in saturated groundwater flows," Rev. Geophys. 44, RG3002 (2006).

[49] S. Torquato, Random Heterogeneous Materials (Springer, 2002).

[50] A. Fiori, I. Janković, and G. Dagan, "Effective conductivity of heterogeneous multiphase media with circular inclusions," Phys. Rev. Lett. (2005).

[51] M. Dentz, P. K. Kang, and T. Le Borgne, "Continuous time random walks for non-local radial solute transport," Adv. Wat. Res. 82, 16-26 (2015).

[52] M. F. Shlesinger, "Asymptotic solutions of continuoustime random walks," J. Stat. Phys. 10, 421-434 (1974).

[53] M.G. McDonald and A.W. Harbaugh, A modular threedimensional finite-difference groundwater flow model. (Washington : Scientific Publications, 1984).

[54] David W. Pollock, "Semianalytical computation of path lines for finite difference models," Ground Water 26, 743750 (1988).

[55] C. Cordes and W. Kinzelbach, "Continuous groundwater velocity fields and path lines in linear, bilinear, and trilinear finite elements," Water Resour. Res. (1992). 